\title{
- Catalytic Mechanism and Allosteric Regulation of UDP-Glucose 2 Pyrophosphorylase from Leishmania major
}

\author{
3 Jana Führing, ${ }^{\dagger}$ Johannes T. Cramer, ${ }^{\dagger}$ Françoise H. Routier, ${ }^{\dagger}$ Anne-Christin Lamerz, ${ }^{\dagger, \dagger}$ Petra Baruch, ${ }^{\dagger}$ \\ ${ }_{4}$ Rita Gerardy-Schahn, ${ }^{\dagger}$ and Roman Fedorov $\$, \S, *$ \\ $5^{\dagger}$ Institute for Cellular Chemistry, OE4330, Hannover Medical School, Carl-Neuberg-Strasse 1, 30625 Hannover, Germany \\ $6^{*}$ Research Division for Structural Analysis, OE8830, Hannover Medical School, Carl-Neuberg-Strasse 1, 30625 Hannover, Germany \\ ${ }_{7}^{\S}$ Institute for Biophysical Chemistry, OE4350, Hannover Medical School, Carl-Neuberg-Strasse 1, 30625 Hannover, Germany
} Supporting Information

9 ABSTRACT: UDP-glucose pyrophosphorylase (UGP) is a nucleotidyl-

10 transferase of central importance in all organisms and considered an 11 attractive drug target in the human pathogens Leishmania and 12 Trypanosoma. Here, we used wild-type and mutant Leishmania major 13 UGP to solve the crystal structures of postreactive, UTP, and UDP-Glc 14 bound states and performed kinetic and theoretical chemistry analysis of 15 the enzymatic reaction. The new data filled critical gaps in the 16 knowledge of the UGP mechanism and allowed reconstructing the 17 complete enzymatic cycle on three levels: global (movements of 18 molecular functional blocks), local (behavior of separate residues), and 19 chemical (quantum mechanical description of enzymatic reaction).

20 Results were integrated into a model of UGP activity describing structural changes along the cycle, the mechanisms of substrate 21 binding, UGP catalysis, and product release. Our study revealed the mechanisms of allosteric regulation common for 22 nucleotidyltransferases and, in particular, the mechanical control of the chemical reaction in the active site.

23 KEYWORDS: nucleotidyltransferase, catalysis, allosteric regulation, molecular mechanism, Leishmania, Trypanosoma,

24 complete enzymatic cycle, mechanochemistry

\section{INTRODUCTION}

26 Enzymes of the large superfamily of nucleotidyltransferases 27 (nucleotide triphosphate (NTP) transferases) catalyze key 28 cellular processes in all kingdoms of life. ${ }^{1}$ Within the wide 29 spectrum of functions, members of this superfamily are 30 responsible for DNA repair, RNA editing, and activation of 31 metabolites used in catabolic pathways, as well as detoxification 32 and antibiotic resistance mechanisms. ${ }^{1}$ Despite large diversity 33 existing at primary and quaternary structure levels (mono-, di-, 34 tetra-, hexa-, and octameric forms have been described ${ }^{2-6}$ ), 35 nucleotidyltransferases commonly adopt Rossmann-like $\alpha / \beta / \alpha$ 36 sandwich folds in their catalytic domains, ${ }^{6}$ require $\mathrm{Mg}^{2+}$ for 37 catalytic activity, ${ }^{7-9}$ and follow a sequentially ordered bibi 38 catalytic mechanism, with the NTP binding first. ${ }^{4,5,10,11}$ In 39 keeping with their central cellular positions, nucleotidyltrans40 ferases have been demonstrated to be a subject of elaborate 41 regulatory mechanisms, ${ }^{1}$ including allosteric effectors, redox 42 regulation, phosphorylation ${ }^{12-15}$ and sequestration of active 43 enzymes by oligomerization. ${ }^{3,16}$ Although intensively studied at 44 both the kinetic and structural levels, ${ }^{4,5,10,11}$ molecular details 45 explaining common functional properties of nucleotidyltrans46 ferases, such as the bibi kinetics and the role of the essential $47 \mathrm{Mg}^{2+}$ ion, are either missing or equivocally discussed., ${ }^{70}$ 48 Similarly, it remains unknown how the various levels of 49 structural organization combine with allosteric regulation to affect the reaction cycles of these enzymes. Elucidating these 50 mechanisms is, however, essential for the fundamental 51 understanding of nucleotidyltransferases and the development 52 of new concepts to specifically inhibit individual members. 53

Recently, we cloned UDP-glucose (UDP-Glc) pyrophos- 54 phorylase from Leishmania major (LmUGP). ${ }^{2}$ This enzyme 55 catalyzes the reversible conversion of glucose-1-phosphate 56 (Glc-1-P) and uridine 5'-triphosphate (UTP) to UDP-Glc 57 and inorganic pyrophosphate $\left(\mathrm{PP}_{\mathrm{i}}\right)$ in the presence of $\mathrm{Mg}^{2+} 58$ ions. Very different from all known pro- and eukaryotic UGPs, 59 the LmUGP is an active monomer with no capability to form 60 higher oligomers that, for example, in plants represent 61 sequestrated enzyme forms. ${ }^{3,16}$ This unique feature together 62 with the fact that UGP is a promising target for the 63 development of specific antiparasitic agents ${ }^{17,18}$ motivated us 64 to investigate the function of this enzyme at the macro- 65 molecular, atomic, and chemical levels.

Previously obtained open and closed structures of LmUGP 67 ( $\mathrm{pdb}$ codes: $2 \mathrm{OEF}$ and $2 \mathrm{OEG}$, respectively) revealed major 68 conformational change between these two states. ${ }^{19}$ To 69 understand these molecular movements and reconstruct a 70

Received: September 4, 2013

Revised: October 30, 2013 


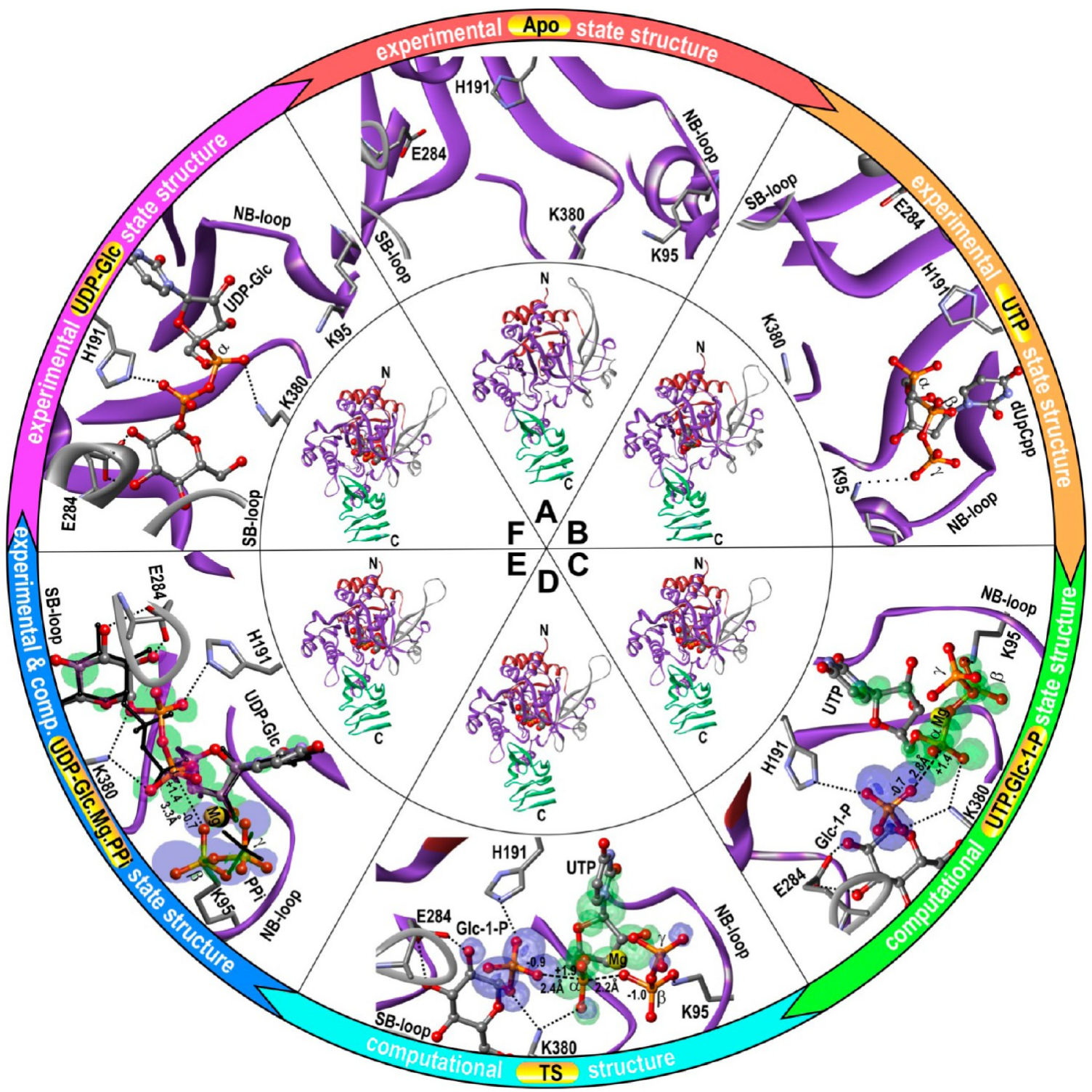

Figure 1. Enzymatic cycle of LmUGP. The inner circle shows the overall views of LmUGP for each state in ribbon representation. The outer circle shows the close-ups of the UGP active site. The N-terminal, C-terminal, and central catalytic domains are shown in red, green, and blue, respectively. The lock region is shown in gray. The substrates are shown in a Corey-Pauling-Koltun representation. The outer segments $\mathrm{C}$, D, and $\mathrm{E}$ contain graphical representations of HOMO (blue) and LUMO (green). Only lobes of the same sign localized on reacting atoms are shown. (A) Open state (apo form) representations based on the experimental structure 2OEF. ${ }^{19}$ The $\mathrm{N}$-terminal/catalytic and C-terminal domains are at their maximum distance, the seven-stranded $\beta$-sheet is relaxed, the lock is open. Only the binding site for UTP is present. (B) Crystal structure of LmUGP-dUpCpp complex representing the UTP state. The seven-stranded $\beta$-sheet and the NB loop acquire intermediate conformations between the open and the closed states, and the lock region remains open. The phosphates are located in the interface between the catalytic and C-terminal domains with the $\gamma$ phosphate stabilizing the NB loop. The binding pocket for the glucose ring is formed. (C) QM/MM model of the prereactive state. The whole structure is in the closed state; both substrates are stabilized in position for the reaction. (D) The trigonal bipyramidal geometry of the transition state. (E) QM/MM (colored) and experimental (black) models of the postreactive state. (F) Experimental structure of UDP-Glc state (2OEG). ${ }^{19}$ The pyrophosphate dissociation leads to the relaxation of the NB loop and the activation of the lock mechanism for opening the LmUGP structure.

71 comprehensive picture of the enzymatic cycle, we aimed to 72 obtain additional intermediate state geometries. Here, we 73 determined the crystal structures of wild-type and mutant 74 LmUGP in the presence of substrates, products, or their 75 analogs.

76 The new structures for the first time revealed the binding 77 sites for all UGP products, elucidated the key role of the UTP 78 nucleoside and $\gamma$-phosphate in UGP activation, and helped 79 explain the allosteric mechanisms that accompany binding and 80 stabilization of both substrates of the bibi reacting LmUGP. 81 Moreover, novel insights into the elements responsible for substrate binding and product release have been obtained. Our 82 structural analysis is supported by original kinetics data 83 presented in this study and by published data for the forward 84 and backward reactions of the wild-type LmUGP and its 85 functional mutants. ${ }^{2,19}$ The ab initio quantum mechanics/ 86 molecular mechanics (QM/MM) calculations of the LmUGP 87 reaction pathway helped elucidate electronic effects and the 88 roles of $\mathrm{Mg}^{2+}$ and active site residues in UGP catalysis.

\section{9}

The resulting model of UGP activity describes structural 90 changes along the catalytic cycle, the mechanisms of substrate 91 binding, UGP catalysis, and product release (Figure 1). With $92 \mathrm{fl}$ 


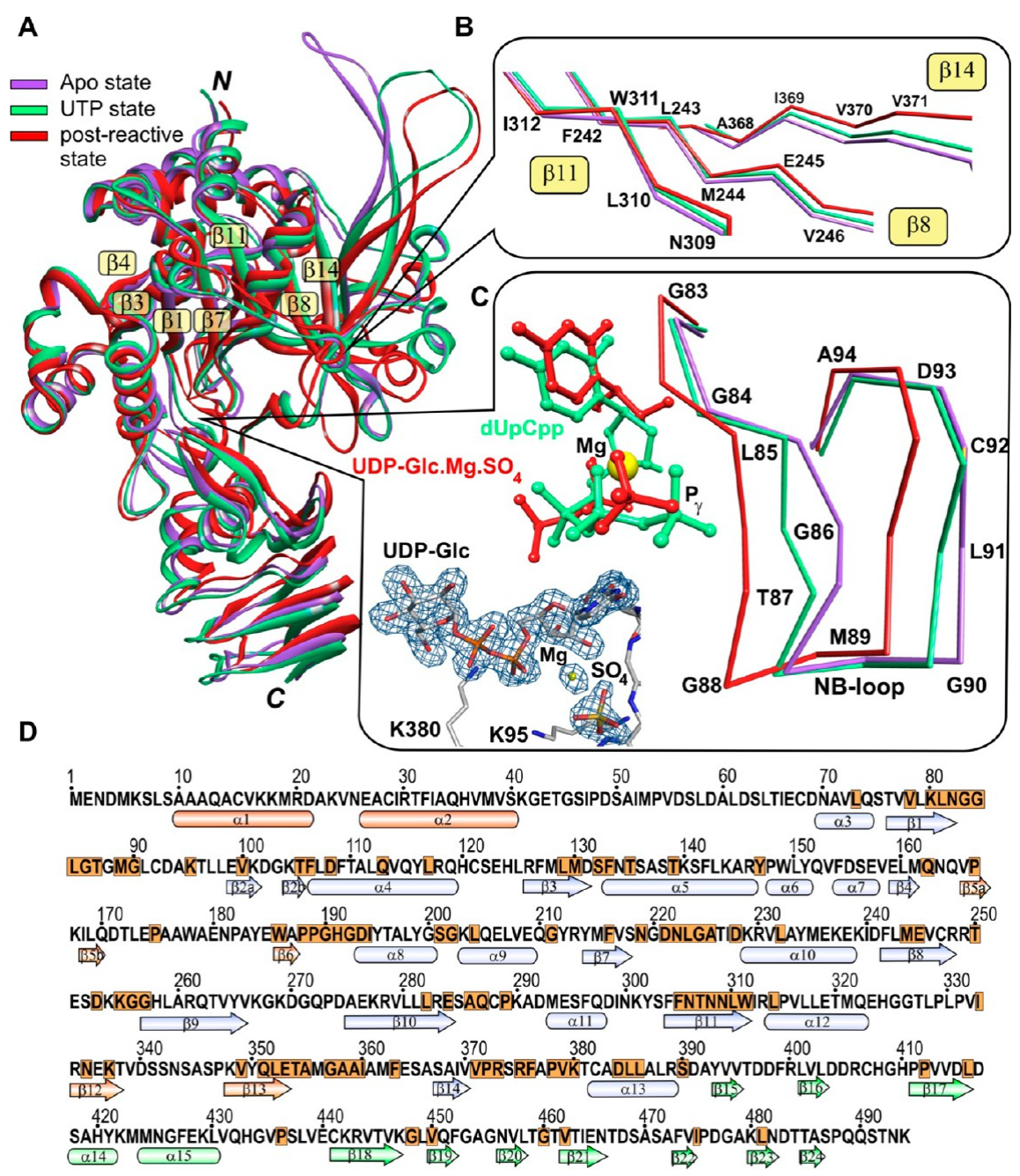

Figure 2. (A) Superposition of the LmUGP structures in the apo state (blue; PDB code: $2 \mathrm{OEF}^{19}$ ), the UTP state (green; LmUGP.dUpCpp complex), and the postreactive state (red; LmUGP.UDP-Glc $\cdot \mathrm{Mg}^{2+} \cdot \mathrm{SO}_{4}$ complex) illustrates the overall conformational changes upon binding the first and second substrates. The torsional deformation of the seven-stranded $\beta$-sheet in the area near the glucose binding site is shown in panel $\mathrm{B}$. Panel $\mathrm{C}$ shows structural changes in the NB loop area and the $F_{\text {obs }}-F_{\text {calc }}$ electron density omit map contoured at $3.0 \sigma$ around the ligands UDP-Glc, $\mathrm{Mg}^{2+}$ and $\mathrm{SO}_{4}{ }^{2-}$ in the wild-type LmUGP postreactive state structure. (D) Amino acid sequence of LmUGP. The secondary structure elements of the N-terminal domain are red; catalytic domain, blue; and C-terminal domain, green. ${ }^{19}$ The residues conserved in UGPs from different organisms are highlighted in orange.

93 this description of the complete reaction cycle of LmUGP, we 94 provide novel insights into the function of nucleotidyltrans95 ferases and a general guide for the in-depth mechanistic analysis 96 of these enzymes. Finally, we demonstrate how allosteric 97 mechanisms control specific recognition of glucose by LmUGP 98 and regulate the chemical reaction in the active site via 99 mechanical force transduction.

\section{EXPERIMENTAL SECTION}

101 Protein Preparation and Crystallization. Untagged and $102 \mathrm{C}$-terminally $\mathrm{His}_{6}$-tagged wild-type L. major UGP were 103 prepared as described by Lamerz et al. ${ }^{2}$ Mutant proteins 104 L281D and H191L were prepared as described by Steiner et $105 \mathrm{al}^{19}$ The E284A mutant was created by overlap extension PCR 106 using mutagenesis primers with the sequences 107 gctgcggGCGtccgc (sense) and gcggaCGCccgcagc (antisense), 108 respectively, in which the mutated codon is given in uppercase. 109 The C-terminally $\mathrm{His}_{6}$-tagged E284A mutant protein was then

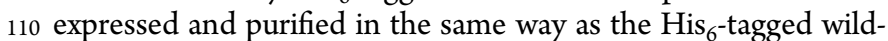
111 type protein. ${ }^{2}$ Crystals of the wild-type enzyme complexes were grown at $20{ }^{\circ} \mathrm{C}$ by vapor diffusion in hanging drop geometry 112 with $500 \mu \mathrm{L}$ of reservoir solution. Crystals of the L281D and 113 the $\mathrm{H} 191 \mathrm{~L}$ mutants were grown at 4 and $20^{\circ} \mathrm{C}$, respectively, in 114 sitting drop geometry with $500 \mu \mathrm{L}$ of reservoir solution. For the 115 UTP state, $1 \mathrm{mM}$ dUpCpp and $4 \mathrm{mM} \mathrm{MgCl}_{2}$ were added to the 116 wild-type protein $(12.4 \mathrm{mg} / \mathrm{mL} ; 10 \mathrm{mM}$ Tris- $\mathrm{HCl}, \mathrm{pH} 7.5 ; 117$ $100 \mathrm{mM} \mathrm{NaCl} ; 2 \mathrm{mM} \mathrm{MgCl}$; 2 mM DTT). A $1.0 \mu \mathrm{L}$ portion 118 of the resulting complex was mixed 1:1 with the reservoir 119 solution (0.1 M MES, pH 6.4; 20\% w/v PEG-4000; $200 \mathrm{mM} 120$ $\mathrm{Li}_{2} \mathrm{SO}_{4}$ ). For the postreactive state, $1.1 \mu \mathrm{L}$ of the wild-type 121 protein $(7 \mathrm{mg} / \mathrm{mL} ; 10 \mathrm{mM}$ Tris- $\mathrm{HCl}, \mathrm{pH} 7.5 ; 100 \mathrm{mM} \mathrm{NaCl} ; 122$ $2 \mathrm{mM} \mathrm{MgCl}$; $2 \mathrm{mM} \mathrm{DTT;} 4 \mathrm{mM}$ UDP-Glc) was mixed 1:1 123 with the reservoir solution $(0.1 \mathrm{M}$ Bis-Tris, $\mathrm{pH} 6.0 ; 22 \% \mathrm{w} / \mathrm{v} 124$ PEG-MME-2000; $\left.20 \mathrm{mM}\left(\mathrm{NH}_{4}\right)_{2} \mathrm{SO}_{4}\right)$. The L281D mutant 125 was concentrated to $2.3 \mathrm{mg} / \mathrm{mL}$ in the same buffer adjusted to 126 $\mathrm{pH}$ 7.8. The reservoir solution contained 0.1 M Bis-Tris, $\mathrm{pH} 127$ 6.6, and 28\% w/v PEG-MME-2000. In the case of the H191L 128 mutant, the protein buffer contained $10 \mathrm{mg} / \mathrm{mL} \mathrm{LmUGP}_{\mathrm{H} 191 \mathrm{~L}} ; 129$ $10 \mathrm{mM}$ Tris-HCl, pH 7.5; $100 \mathrm{mM} \mathrm{NaCl} ; 2 \mathrm{mM} \mathrm{MgCl}_{2} ; 2130$ $\mathrm{mM}$ DTT; $4 \mathrm{mM}$ UTP; and $4 \mathrm{mM}$ Glc-1-P. The reservoir 131 
132 solution contained $10 \% \mathrm{v} / \mathrm{v}$ glycerol; $0.05 \mathrm{M}$ citrate buffer, $\mathrm{pH}$ 133 5.2; and 1.1 M sodium citrate. Prior to flash-cooling, all crystals 134 were rinsed in reservoir solution supplemented with $20 \%$ 135 ethylene glycol for cryoprotection.

136 In Vitro Activity Measurements and Kinetic Data 137 Analysis. To calculate reaction energy parameters, the in vitro 138 activity of wild-type LmUGP was measured at 25 and $37{ }^{\circ} \mathrm{C}$ 139 using the untagged recombinant protein. In the forward 140 direction of the reaction (production of UDP-Glc), formation 141 of pyrophosphate was continuously detected using the 142 EnzChek Pyrophosphate Assay Kit (Molecular Probes) in a 143 buffer containing $50 \mathrm{mM}$ Tris $/ \mathrm{HCl}, \mathrm{pH} \mathrm{7.8}$, and $10 \mathrm{mM}$ $144 \mathrm{MgCl}_{2}$. UTP was kept at a saturating concentration of $1 \mathrm{mM}$, 145 and the concentration of Glc-1-P was varied between 0.1 and 3 $146 \mathrm{mM}$. The backward reaction (formation of UTP) was 147 monitored using the continuous assay described by Damerow 148 et al. ${ }^{20}$ Here, UDP-Glc was supplied at a saturating 149 concentration of $1 \mathrm{mM}$, and pyrophosphate concentration 150 was varied between 0.1 and $2 \mathrm{mM}$. For the forward and 151 backward reaction activity assays, a $1.84 \mathrm{mg} / \mathrm{mL}$ UGP stock 152 was diluted 1:40000 and 1:10000, respectively. The reaction 153 was started by addition of $10 \mu \mathrm{L}$ of diluted UGP unto a final 154 reaction volume of $100 \mu \mathrm{L}$. The resulting values of $[\mathrm{E}]_{0}$ for the 155 forward and backward reactions were $8.6915 \times 10^{-11} \mathrm{M}$ and $1563.4766 \times 10^{-10} \mathrm{M}$, respectively. Product formation was 157 continuously monitored at $360 \mathrm{~nm}$ in a microplate reader 158 (Power-Wave $340 \mathrm{KC} 4$ system, Bio-Tek). The rates of product 159 release were plotted against the varied substrate concentration, 160 and $K_{\mathrm{m}}$ and $V_{\max }$ were calculated using the nonlinear fitting 161 function in PRISM software (GraphPad Software, Inc.). The 162 free-energy barriers resulting from the experimental $V_{\max }$ values 163 (Supporting Information Figure S2A,B) were calculated 164 assuming that the reaction catalyzed by LmUGP proceeds via 165 the sequentially ordered bibi catalytic mechanism common for 166 nucleotidyltransferases, with a single barrier between the pre167 and postreactive states. In this case, the rate-limiting step 168 constant corresponding to the substrate-to-product conversion 169 is identical to $k_{\text {cat }}=V_{\max } /[\mathrm{E}]_{0}$, and the reaction free energy 170 barrier can be estimated according to the transition state theory 171 (TST) as $\Delta G^{\#}=-R T \ln \left(k_{\text {cat }} h\right) /\left(k_{\mathrm{B}} T\right)$, where $R, k_{\mathrm{B}}$, and $h$ are 172 the ideal gas, Boltzmann's, and Planck's constants, respectively, 173 and $T$ is the absolute temperature.

174 To compare kinetic parameters of the LmUGP E284A 175 mutant and wild-type LmUGP (both C-terminally His-tagged), 176 activity measurements at $25{ }^{\circ} \mathrm{C}$ in the forward direction of the 177 reaction were carried out and evaluated as described above, 178 with the following modifications: UTP concentration was 179 varied between 0.05 and $1 \mathrm{mM}$ at a saturating supply of $2 \mathrm{mM}$ 180 Glc-1P, and titration of Glc-1P between 0.05 and $2 \mathrm{mM}$ was 181 performed at a saturating concentration of $1 \mathrm{mM}$ UTP.

182 Diffraction Data Collection and Structure Determi183 nation. Diffraction data were collected at the EMBL beamlines $184 \mathrm{X} 11$ and X13 at the DORIS storage ring, DESY, Hamburg, and 185 the beamlines ID23-2 and ID29, at ESRF, Grenoble, using 186 MAR CCD and Dectris Pilatus $6 \mathrm{M}$ detectors (see Supporting 187 Information Table S1 for details), and reduced with the XDS 188 program package. ${ }^{21}$ The structures of $\mathrm{LmUGP}_{\mathrm{wt}} \cdot \mathrm{dUpCpp}$ 189 complex and LmUGP ${ }_{\mathrm{H} 191 \mathrm{~L}}$ mutant was solved by molecular 190 replacement (CCP4 software suite ${ }^{22,23}$ ) using the structure of 191 LmUGP apo form (pdb code: $2 \mathrm{OEF}^{19}$ ) as an initial model. 192 Structure determination of $\mathrm{LmUGP}$ wt $\mathrm{UDP}-\mathrm{Glc} \cdot \mathrm{Mg}^{2+} \cdot \mathrm{SO}_{4}$ and $193 \mathrm{LmUGP}_{\mathrm{L} 281 \mathrm{D}}$.UDP-Glc complexes was initiated by a round of $194 \mathrm{CNS}^{24,25}$ rigid body refinement using the protein part of the
LmUGP coordinates for the closed conformation of the 195 enzyme (pdb code: $2 \mathrm{OEG}^{19}$ ) as an initial model. Refinement 196 was continued with CNS, SHELX, ${ }^{26}$ and REFMAC ${ }^{27}$ and 197 included simulated annealing and individual B-factor refine- 198 ment. The initial electron density maps were calculated using 199 ARP/wARP. ${ }^{28}$ During cyclic rounds of refinement and manual 200 rebuilding using the $\mathrm{COOT}^{29}$ program, ligands and solvent 201 molecules were included in the models (see Figures 2C and $202 \mathrm{f} 2$ Supporting Information S1A-D). The final models displayed 203 good stereochemistry (see Supporting Information Table S1). 204 Structure comparisons were performed using SSM and LSQ 205 superpose tools of COOT.

Molecular Dynamics Simulations. To study the effect of 207 the E284-glucose interaction on the conformation of LmUGP, 208 molecular dynamics (MD) simulations were performed using 209 the $\mathrm{CNS}^{24,25}$ program package. The starting geometry was 210 obtained from the coordinates of the $\mathrm{LmUGP}_{\mathrm{wt}} \cdot \mathrm{dUpCpp} 211$ complex (UTP state) and the glucose coordinates from the 212 superimposed structure of the $\mathrm{LmUGP} \mathrm{wt}_{\mathrm{w}} \cdot \mathrm{UDP}-\mathrm{Glc} \cdot \mathrm{Mg}^{2+} \cdot \mathrm{SO}_{4} 213$ complex (postreactive state). In this geometry, the distances 214 between the oxygen atoms of the E284 carboxyl group and the 215 $2^{\prime}, 3^{\prime}$-oxygen atoms of glucose were close to $6 \AA$. At the start of 216 the simulations, the hydrogen bond distance restraints between 217 these atoms were assigned. The Cartesian MD simulations were 218 performed for $1 \mathrm{~ns} \mathrm{MD}$ trajectory at a constant temperature of 219 $300 \mathrm{~K}$. At the end of the dynamic trajectory, the model 220 geometry was optimized using CNS-DEN refinement ${ }^{30}$ without 221 experimental energy terms.

Quantum Chemical Modeling of LmUGP Reaction 223 Pathway. The QM/MM simulations of the LmUGP reaction 224 pathway were performed using the Firefly Quantum Chemistry 225 package, ${ }^{31}$ based in part on the GAMESS (US) ${ }^{32}$ source code. 226 The QM subsystem included the substrates, water, $\mathrm{Mg}^{2+}$, and 227 fractions of the side chains for the protonated residues K95, 228 $\mathrm{H} 191$, and K380. It contained 94 atoms in total and had a 229 charge of -1 . The rest of the protein was included into the MM 230 part. Geometry optimizations, Hessian calculations, saddle 231 point location, and intrinsic reaction coordinate (IRC) 232 simulations in the QM part were carried out using density 233 functional theory (DFT) with the B3LYP hybrid func- 234 tional, $^{33,34}$ Vosko-Wilke-Nusair electron gas formula 5235 correlation, ${ }^{35,36}$ and the valence double- $\zeta$ polarized basis set 236 $6-31 \mathrm{G}^{*}$ for all atoms. The MM3 force field parameters ${ }^{37-39} 237$ were used for the MM part. The starting geometry for the 238 saddle point location search was generated from the 239 coordinates of the postreactive state complex and the substrate 240 coordinates from the structure of LmUGP.dUpCpp complex 241 obtained in this work. This structure was optimized using an 242 unconstrained QM/MM geometry minimization procedure. 243

The search for the saddle point location was started by 244 successively decreasing the distance between the reacting atoms 245 $\mathrm{O}_{3 \mathrm{P}}{ }^{\text {Glc-1-P }}$ and $\mathrm{P}_{\alpha}{ }^{\mathrm{UTP}}$ in the direction of the forward reaction and 246 performing constrained geometry minimization at each step. 247 Upon reaching the flat conformation of the $\mathrm{P}_{\alpha} \mathrm{O}_{3}$ group of 248 UTP, the precise location search of the saddle point was 249 performed using a quadratic approximation augmented Hessian 250 technique. ${ }^{40-42}$ The transition state geometry was located at a 251 point with a single imaginary frequency of Hessian. After 252 finding the transition state, the structures of the pre- and 253 postreactive states were obtained by descent forward and 254 backward from the saddle point along the steepest descent path 255 in mass-weighted Cartesian coordinates using the IRC 256 method. ${ }^{43}$ The resulting models of pre- and postreactive states 257 
258 corresponded to the true minima on the potential energy 259 surface with all real vibrational frequencies. These structures 260 were used for further analysis and comparison with 261 experimental data. The free energy barriers for forward and 262 backward reactions were calculated using harmonic normal 263 mode approximation.

\section{$264 \square$ RESULTS}

265 LmUGP.dUpCpp Complex and the Mechanism of UTP 266 Binding. To study the effect of UTP-binding on the structure 267 and function of LmUGP, we crystallized LmUGP in the 268 presence of the nonreactive UTP analog dUpCpp (see 269 Experimental Section for details). The structure solved by 270 molecular replacement and refined to 3.0 Å resolution showed 271 good electron density for the UTP analog bound to the active 272 site (Supporting Information Figure S1C). The overall 273 conformation of the enzyme is more similar to the open ${ }^{19}$ 274 than to the closed (postreactive; see below) state, with several 275 regions acquiring intermediate conformations (Figure 2A). 276 Compared with the open state, the large magnitude change is 277 located in the nucleotide-binding (NB) loop, which shifts $2.4 \AA$ 278 toward the phosphate moiety, bounding the nucleotide pocket 279 (Figure $2 \mathrm{C}$ ). The seven-stranded $\beta$-sheet $(\beta 1, \beta 3, \beta 4, \beta 7, \beta 8$, $280 \beta 11, \beta 14$; Figure 2D) undergoes a torsional deformation, where 281 strands $\beta 1, \beta 7, \beta 8$ (and adjacent region of the helix $\alpha 10$ ), $\beta 11$, 282 and $\beta 14$ shift toward their positions in the closed state (Figure $2832 \mathrm{~B}$ ), while the conformation of $\beta 3$ and $\beta 4$ remains close to the 284 open state. The nucleoside is coordinated by residues L81, 285 G83, G84 (NB loop), M130 ( $\beta 3$ ), Q162 (end of $\beta 4$ ), G190$286 \mathrm{H} 191$ (loop $\beta 6-\alpha 8$ ), and N219-D221 (end of $\beta 7$ ). Most of 287 these residues remain structurally conserved in the open, UTP, 288 and closed states of the enzyme, which explains why UTP can 289 bind to the apo form and activate the conformational transition. 290 To understand the role of nucleoside binding in LmUGP 291 activation, we crystallized the inactive LmUGP mutant $292 \mathrm{H}_{191 \mathrm{~L}^{19}}$ in the presence of both UTP and Glc-1-P, but no 293 electron density was observed for the substrates. Instead, the 294 mutant structure revealed that the side chain of L191 occupied 295 part of the space necessary for the nucleoside binding (Figure 296 3C). As a result, UTP could not bind, and the mutant 297 conformation remained very close to the open form of the wild298 type protein. This finding supported the primary role of the 299 nucleoside binding in UGP activation.

300 In the X-ray structure of the LmUGP.dUpCpp complex, the 301 phosphates of the UTP analog were coordinated by the 302 residues in the interface between the catalytic and C-terminal 303 domains. It is interesting that in this state, the strictly conserved 304 residue K380, known to interact with the product UDP-Glc and 305 to be essential for enzymatic activity, ${ }^{19}$ is located $8 \AA$ away from 306 the $\alpha$-phosphate and does not make any interaction with the 307 phosphate moiety (Figure 1B). The $\gamma$-phosphate forms three 308 hydrogen bonds and several van der Waals interactions with the 309 NB loop residues G84-T87 and a salt bridge with the strictly 310 conserved K95. These interactions explain the large shift of the 311 NB loop upon UTP binding. As the NB loop, in turn, is bound 312 to the C-terminal domain via a hydrogen bond and extensive 313 hydrophobic contacts, the new structural data elucidate the role 314 of the $\gamma$-phosphate in the mechanism of UGP activation.

315 Glucose-1-phosphate Binding to LmUGP. As typical in 316 enzymes with sequentially ordered bibi kinetics, the binding site 317 for Glc-1-P does not exist in the apo form of UGP. The 318 residues $\mathrm{N} 219(\beta 7), \mathrm{N} 306-\mathrm{N} 308(\beta 11)$, and F376 (link $319 \beta 14-\alpha 13)$, identified as responsible for glucose binding in the

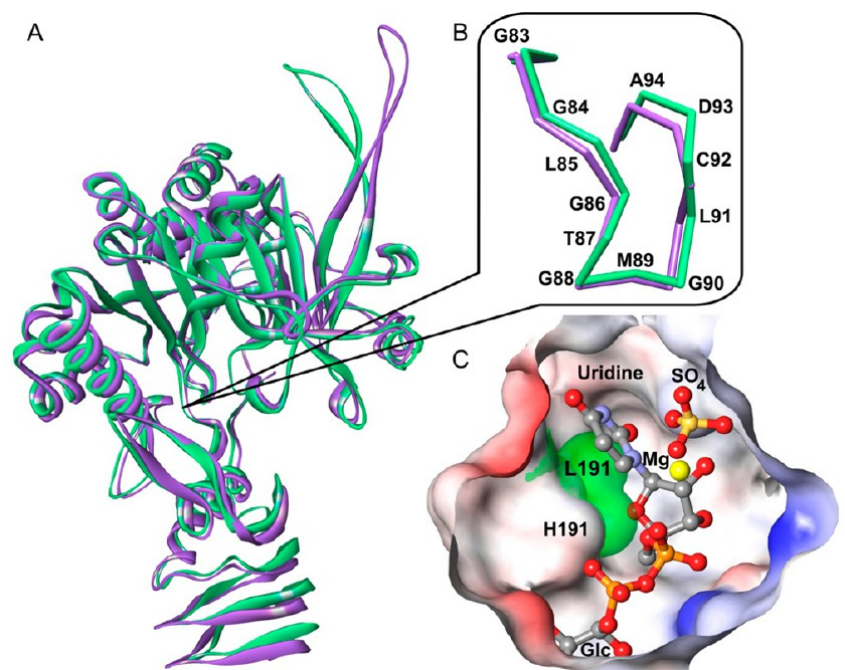

Figure 3. Structural effects of H191L mutation. Superposition of the wild-type LmUGP structure in the apo state (pdb code: $2 \mathrm{OEF}{ }^{19}$ green) and LmUGP ${ }_{\mathrm{H} 191 \mathrm{~L}}$ mutant crystallized in the presence of both substrates (purple) shown for the whole enzyme (A) and for the NB loop area (B). (C) Superposition of the substrate binding pocket in the postreactive state of $\mathrm{LmUGP}_{\mathrm{wt}}$ and in the $\mathrm{LmUGP}_{\mathrm{H} 191 \mathrm{~L}}$ mutant structure. The color scheme of the wild-type protein surface corresponds to the atom charge (blue, positive; red, negative; white, neutral). The van der Waals surface of L191 from the superimposed LmUGP $_{\mathrm{H} 191 \mathrm{~L}}$ mutant structure is shown in green.

closed UGP structure, ${ }^{19}$ overlap with the atoms of the glucose 320 ring in both our postreactive state and UDP-Glc ${ }^{19}$ complexes. 321 In the UTP state, these residues are shifted deeper into the 322 catalytic domain as a result of the torsional deformation of the 323 seven-stranded $\beta$-sheet, which removes steric hindrances and 324 creates a complementary surface for the glucose ring. This, 325 together with the fact that Glc-1-P binding cannot occur 326 without UTP binding first, as demonstrated by STD-NMR 327 studies, ${ }^{2}$ implies that the binding of the glucose ring must play a 328 primary role in the binding of Glc-1-P. Thus, the UTP-induced 329 deformation of the $\beta$-sheet is an important determinant of the 330 Glc-1-P binding site formation.

331

Other structural elements known to contribute to the glucose 332 binding in the above-mentioned closed UGP structures include 333 the highly conserved sugar-binding (SB) loop and the strictly 334 conserved residue E284 located at the end of $\beta 10$ (Figure 2D). 335 These residues belong to a larger region R249-Y302 336 comprising the SB loop, the adjoining $\beta$-hairpin $\beta 9-\beta 10337$ (handle) and helix $\alpha 11$. Superposition of LmUGP structures 338 showed that this region changes dramatically between the open 339 and closed states (Figure 2A). Surprisingly, in the UTP-state 340 structure, the conformation of this region remains almost 341 identical to the open state, with the exception of the top part of 342 the handle, which is known to be flexible, as indicated by the 343 experimental electron density.

In the UTP state, both the SB loop and E284 are distanced 345 from the glucose binding site, but E284 is located $2.0 \AA$ closer 346 than the nearest SB loop residue and, therefore, can bind first. 347 To study the structural effect of the interaction between E284 348 and the glucose ring, we have performed molecular dynamics 349 simulations (see Experimental Section for details). As a starting 350 model, we used the structure of the $\mathrm{LmUGP}_{\mathrm{wt}} \cdot \mathrm{dUpCpp} 351$ complex (UTP state) and the glucose coordinates from the 352 superimposed structure of the $\mathrm{LmUGP}$ wt $\mathrm{UDP}-\mathrm{Glc} \cdot \mathrm{Mg}^{2+} \cdot \mathrm{SO}_{4} 353$ complex (closed postreactive state, see below). During these 354 
355 simulations, the force was applied to the side chain of E284 to 356 bring it to its position in the closed state of the enzyme. E284 is 357 linked to the SB loop via the $\beta$-hairpin structure of the handle 358 and our molecular dynamics simulations suggested that pulling 359 E284 in the direction of the glucose-binding site results in the 360 movement of the whole region R249-Y302 toward its 361 conformation in the closed state.

362 To test this hypothesis, we first examined the role of E284 in 363 glucose binding. Therefore, mutant E284A was constructed and 364 kinetically characterized in comparison with the wild-type 365 enzyme (Supporting Information Figure S2C,D). The specific 366 activity of the mutant E284A was reduced to less than $0.05 \%$ of 367 the wild-type. By measuring $K_{\mathrm{m}}$ values, the affinity of the E284A 368 mutant for UTP was found to be nearly unchanged (E284A, $36973.9 \pm 5.8 \mu \mathrm{M}$ versus wt, $101.2 \pm 7.6 \mu \mathrm{M})$, whereas the affinity 370 for Glc-1-P was drastically impaired (E284A, $977.4 \pm 99.5 \mu \mathrm{M}$ 371 versus wt, $152.8 \pm 14.3 \mu \mathrm{M})$.

372 To study the interaction between E284 and the SB loop, we 373 investigated structural effects of the L281D mutation located in 374 the link connecting these two areas, at the end of the strand $375 \beta 10$. L281 forms a part of the hydrophobic core stabilizing the 376 base of the handle and its contact with the $\mathrm{N}$-terminal and 377 catalytic domains. The L281D mutation leads to a drop of 378 specific activity (measured in the forward reaction) to $16.3 \%$ of 379 the wild type. ${ }^{19}$ Because the residue is spatially remote from 380 active site and SB loop, the mechanism of this inactivation was 381 previously unclear. The crystal structure of the L281D mutant 382 in complex with UDP-Glc solved in this study now shows that 383 the mutation leads to a disruption of the $\beta$-structure of the 384 handle (Figure 4A) and an increase in its flexibility, reflected in 385 the weakening of experimental electron density for this region. 386 In the mutant structure, the interactions between E284 and 387 glucose are not changed, but the distances between E284 and 388 the SB loop or $\alpha 11$ are increased, and a lack of closure is 389 observed in both the SB loop and the $\alpha 11$ area (Figure 4B). 390 Thus, the disruption of the $\beta$-structure caused by the L281D 391 mutation leads to the loss of stiffness in the handle and, 392 therefore, a reduction of its efficiency in transducing tensile 393 force to the SB loop and $\alpha 11$. This, in its turn, leads to a lack of 394 closure in these areas (Figure 4B) and a decrease in the specific 395 activity of the L281D mutant to $16.3 \%$ of the wild-type 396 enzyme. ${ }^{19}$ The top of the handle features significant conforma397 tional flexibility observed in the crystal structures of LmUGP, 398 and its length varies between species. This, in conjunction with 399 the observed effect of the L281D mutation, implies that the 400 residues involved in transduction of the tensile force are located 401 at the base of the handle.

402 Comparison of the UTP state and closed postreactive state 403 structures showed that closing the region R249-Y302 brings 404 the strictly conserved residues H191 and K380 in a favorable 405 position for binding Glc-1-P phosphate and leads to adjust406 ments in the glucose binding site that further increase its 407 complementarity. After closing, the region R249-Y302 is 408 additionally stabilized by a hydrogen bond between the SB loop 409 E251 and the C-terminal domain R443 observed in the closed 410 state structures.

411 Product Formation and Allosteric Regulation of 412 Product Release. By cocrystallizing LmUGP with UDP-Glc, $413 \mathrm{Mg}^{2+}$, and $\mathrm{SO}_{4}{ }^{2-}$ ions, the postreactive state of the enzyme 414 could be trapped, and the structure, resolved to the high 415 resolution of $1.6 \AA$. The postreactive state is the most compact 416 of all known published ${ }^{19}$ and herein presented structures of 417 LmUGP. Both the accessible surface area of the protein and the
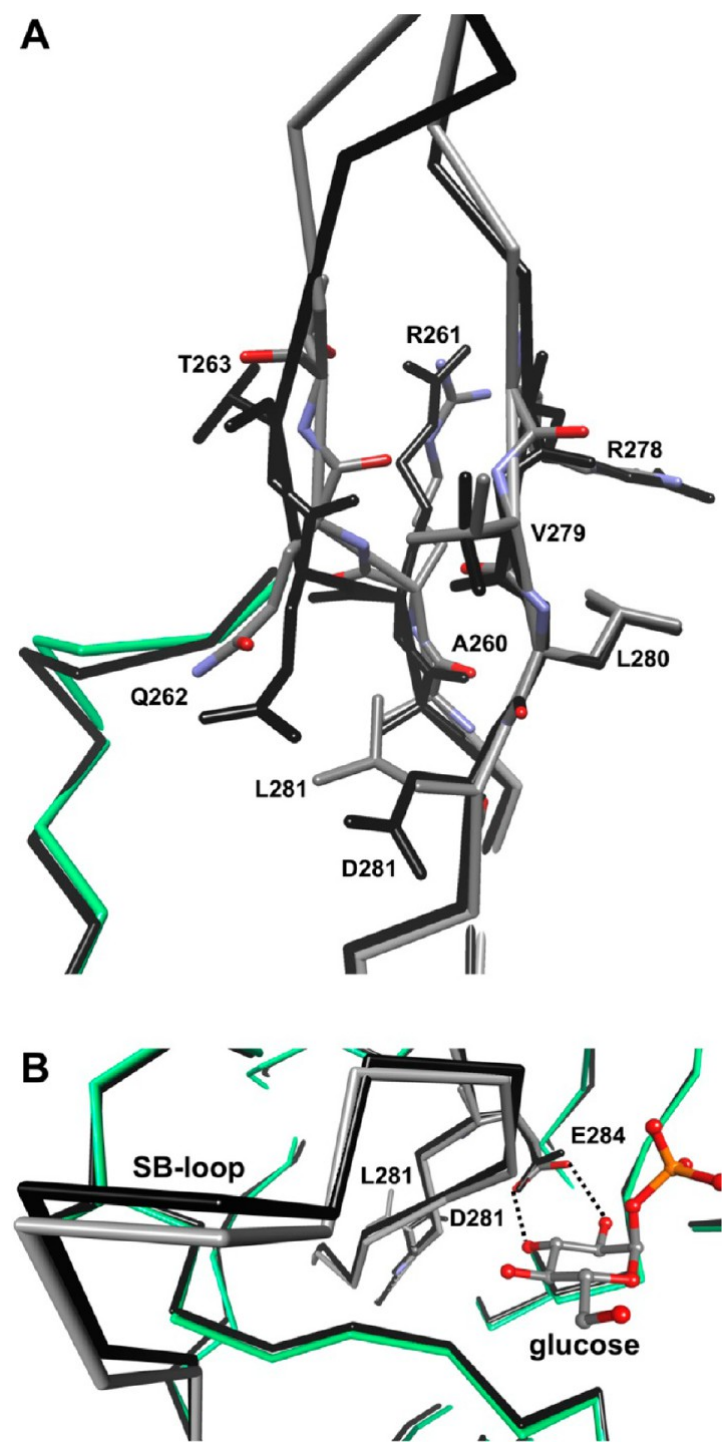

Figure 4. Superpositions of the wild-type (colored) and L281D mutant (black) LmUGP.UDP-Glc complexes. The mutation causes disturbances of the $\beta$-structure at the base of the handle (A), which results in the lack of closure in the SB loop region (B).

distances between key structural elements and the catalytic 418 center were at their minimum in this state, whereas the 419 torsional deformation of the $\beta$-sheet increased (Figure 2B). 420 Compared with the UTP-state structure, the NB loop is shifted 421 $\sim 2$ A closer toward the phosphate moiety, further bounding the 422 nucleotide pocket (Figure 2C). The region R249-Y302 is in its 423 closed state, providing maximal stabilization to the glucose ring. 424 The amino group of the strictly conserved K380 is moved $5.6 \AA 425$ toward the nucleotide, and an $\mathrm{H}$-bond with the $\alpha$-phosphate of 426 UDP-Glc is formed. In the crystal structure, the sulfate ion 427 occupies the position of the $\gamma$-phosphate in the LmUGP. 428 $\mathrm{dUpCpp}$ complex. The identified magnesium ion coordinated 429 sulfate, $\alpha$-phosphate, and a water molecule. This water occupied 430 the space between the $\alpha$ - and $\gamma$-phosphates and was additionally 431 coordinated by an H-bond and electrostatic interactions with 432 G86 and K95 of the NB loop. Superposition of the UTP and 433 postreactive state structures suggested that in the prereactive 434 state, the magnesium should be coordinated between the $\alpha$ - 435 and $\beta$-phosphates of UTP (Supporting Information Figure S4). 436 Of note, with the crystal structure of the postreactive state of 437 
438 LmUGP, we present the first experimental structure in which 439 the binding sites of all UGP products could be identified.

440 Comparison of the crystal structures of LmUGP in the 441 postreactive and the UDP-Glc-bound states revealed that 442 dissociation of the pyrophosphate and magnesium leads to a 443 significant change in the NB loop conformation (Figure 5). The

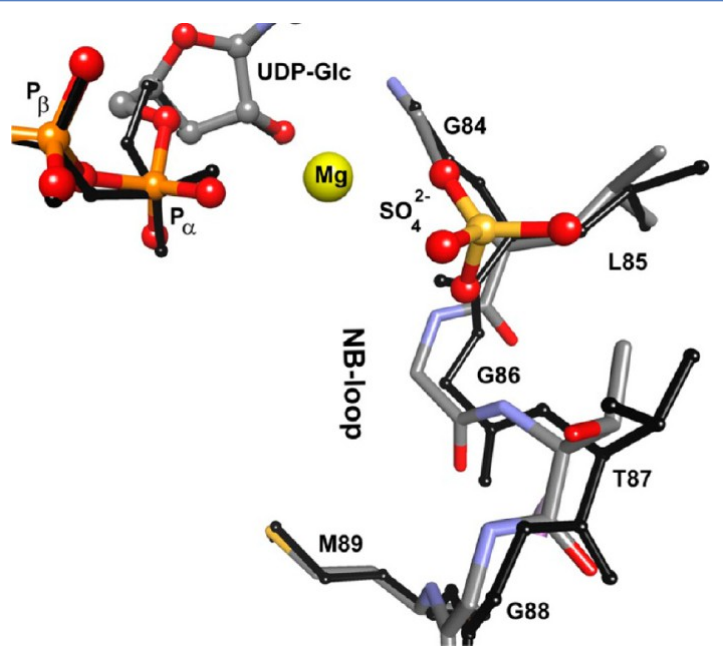

Figure 5. Experimental LmUGP crystal structures representing the postreactive (colored) and UDP-Glc (black) states. The superposition reveals conformational changes of the NB loop and phosphate moiety of UDP-Glc caused by the dissociation of pyrophosphate and $\mathrm{Mg}^{2+}$.

444 G84-G88 residues and the adjacent G90-D93 region moved 445 away from the active site, making it less compact. Because of 446 the loss of the hydrogen bond with the $\gamma$-phosphate, the 447 carbonyl group of the L85 peptide bond was flipped in the 448 UDP-Glc state. As a result, the NB loop acquired an 449 intermediate conformation between those of postreactive and 450 apo states. In the crystal structure of the UDP-Glc state, the 451 experimental electron density for this region was weaker and 452 more disordered than that in the postreactive state $(20 \%$ 453 decrease in the signal-to-noise ratio for $\mathrm{C}_{\alpha}$ atoms). A similar 454 effect was observed for the SB loop residues, where 455 destabilization led to a partial opening of the structure in the 456 area of helix $\alpha 11$ (residues M293-N300) located at the end of 457 the region $\mathrm{R} 249-\mathrm{Y} 302$. This destabilization effect is propagated 458 from the NB to the SB loop via the shift of G88, which moves 459 the conserved K422 (C-terminal domain) closer toward K255 460 (SB loop) and, thus, increases the electrostatic repulsion 461 between the two regions.

462 The flexible top part of the handle, including residues Y265$463 \mathrm{~K} 277$ that are located farther away from the protein globule and 464 not stabilized by intramolecular interactions, is differently 465 structured in the postreactive and the UDP-Glc states. The lack 466 of electron density for $\mathrm{Mg}^{2+}$ in the UDP-Glc complex suggests 467 that the ion dissociates together with the pyrophosphate. The 468 phosphate moiety of UDP-Glc also undergoes a conformational 469 change upon dissociation of $\mathrm{Mg}^{2+}$ and the $\mathrm{PP}_{\mathrm{i}}$ (Figure 5). In 470 the UDP-Glc state, the $\mathrm{C}^{*}-\mathrm{O} 5^{*}$ bond connecting the $\alpha$ 471 phosphate to the glucose ring is flipped $80^{\circ}$ in comparison with 472 the experimental geometry of the postreactive state. This 473 change induces a shift of the K380 side chain, resulting in an 474 increased distance between K380 and the oxygens of the $\alpha$ 475 phosphate and, thus, in the drop of affinity for UDP-Glc. All of 476 the above effects reveal the mechanism of unlocking the enzyme structure, which begins with the dissociation of the 477 pyrophosphate.

Quantum Chemical Studies of LmUGP Catalysis. To 479 study the electronic effects of UGP catalysis and identify the 480 role of the active site residues in this process, we performed 481 quantum chemical (QC) calculations of the LmUGP reaction 482 pathway using the IRC procedure. The starting geometry 483 approximation for the saddle point location search was 484 generated using the coordinates of the protein part, the glucose 485 ring, $\mathrm{Mg}^{2+}$ and water from the postreactive state complex, and 486 the substrate coordinates from the structure of LmUGP. 487 dUpCpp complex obtained in this work. After finding the 488 transition state, the models of the pre- and postreactive states 489 were obtained by descent forward and backward from the 490 saddle point along the steepest descent path in mass-weighted 491 Cartesian coordinates using the IRC method. ${ }^{43}$ The overall 492 protein conformation, the local structure of the active site 493 residues, and the positions of nucleoside and glucose ring in the 494 theoretical model of the LmUGP.UTP.Glc-1-P complex are 495 very close to the experimental geometry of the postreactive 496 state (r.m.s.d. = $1.2 \AA$ \&; Figure 1C). This is in agreement with 497 the principle of least motion in chemical reactions. ${ }^{44}$ In the 498 model, the phosphate moiety of UTP is structured similarly to 499 that of the crystal structure of the LmUGP.dUpCpp complex 500 (r.m.s.d. = $0.3 \AA$ ), whereas the phosphate group of Glc-1-P is 501 oriented toward the $\alpha$-phosphate of UTP, with a closest 502 distance of $2.8 \AA$ between the reagents. H191 and K380 503 stabilize the orientation of the Glc-1-P phosphate with 504 hydrogen bonds, ensuring an optimal position of the attacking 505 oxygen with respect to the $\alpha$-phosphate of UTP. In this 506 position, the highest occupied molecular orbital (HOMO) 507 localized on the attacking oxygen $\mathrm{O}_{3 \mathrm{p}}$ of Glc-1-P overlaps with 508 the lowest unoccupied molecular orbital (LUMO) localized on 509 the $\mathrm{P}_{\alpha}$ atom of UTP, providing an electron exchange between 510 the reactants (Figure 1C). Atomic partial charges resulting from 511 Mulliken population analysis ${ }^{45}$ reveal a significant electrostatic 512 component to this interaction.

Importantly, the QC analysis showed that the magnesium ion 514 coordinated by the oxygen atoms of the UTP $\alpha$-phosphate 515 affects both the partial charge distribution between the reacting 516 atoms and the local geometry of the reaction center. 517 Comparison of the QC prereactive state models with and 518 without $\mathrm{Mg}^{2+}$ revealed that the Mulliken charges on $\mathrm{P}_{\alpha}{ }^{\text {UTP }}$ and 519 $\mathrm{O}_{3 \mathrm{P}}{ }^{\text {Glc-1-P }}$ change from +1.4 and -0.7 in the presence of $\mathrm{Mg}^{2+}$ to 520 +1.15 and -0.6 in the absence of $\mathrm{Mg}^{2+}$, respectively. Thus, 521 $\mathrm{Mg}^{2+}$ increases the polarization and electrostatic attraction of 522 the reacting atoms. The involvement of $\mathrm{O}_{1 \alpha}$ and $\mathrm{O}_{3 \alpha}$ of the 523 UTP $\alpha$-phosphate in the coordination sphere of $\mathrm{Mg}^{2+}$ imposes 524 a flattening effect of $10^{\circ}$ on the $\alpha$-phosphate geometry, 525 rendering it closer to the transition state. In the presence of 526 $\mathrm{Mg}^{2+}$, the covalent bond $\mathrm{P}_{\alpha}{ }^{\text {UTP }}-\mathrm{O}_{3 \alpha}{ }^{\text {UTP }}$ of the leaving group is 527 elongated by $0.2 \AA$ compared with that of the $\mathrm{Mg}^{2+}$-free 528 prereactive state, making dissociation along this bond easier. In 529 contrast, however, the positioning, mutual orientation, and 530 overall conformation of the reagents do not depend on $\mathrm{Mg}^{2+}{ }_{531}$ but are defined primarily by the protein matrix.

These data explain why in previous studies $\mathrm{Mg}^{2+}$ was shown 533 to have a pronounced activation effect on UGPs $^{9}$ but is 534 dispensable for nucleotide binding. ${ }^{46}$ Analysis of the potential 535 energy profile along the UGP reaction coordinate revealed one 536 barrier between the pre- and postreactive states. On the top of 537 the barrier, the UTP $\alpha$-phosphate acquires a trigonal 538 bipyramidal configuration with a planar $\mathrm{P}_{\alpha} \mathrm{O}_{3}$ group and the 539 
540 axial coordination sites occupied by the bridging $\mathrm{P}_{\alpha}-\mathrm{O}_{3 \alpha}-\mathrm{P}_{\beta}$ 541 oxygen of UTP and the attacking oxygen from Glc-1-P 542 phosphate (Figure 1D). The angle $\mathrm{O}_{3 \mathrm{P}}{ }^{\text {Glc-1-P }}-\mathrm{P}_{\alpha}{ }^{\text {UTP }}-\mathrm{O}_{3 \alpha}{ }^{\text {UTP }}$ 543 is increased from $160^{\circ}$ in the prereactive state to $167^{\circ}$ in the 544 transition state. The movement of the attacking oxygen toward ${ }_{545} \mathrm{P}_{\alpha}{ }^{\text {UTP }}$ is accomplished via rotational degrees of freedom of the 546 Glc-1-P phosphate group. The elongation of the $\mathrm{P}_{\alpha}{ }^{\text {UTP }}-\mathrm{O}_{3 \alpha}$ UTP 547 bond leads to a shift of the $\mathrm{P}_{\beta}-\mathrm{P}_{\gamma}$ phosphates and $\mathrm{Mg}^{2+}$ toward 548 the NB loop, inducing a small adjustment of the protein main 549 chain around G86. K380, coordinating both Glc-1-P phosphate 550 and UTP $\alpha$-phosphate, shifts in the transition state toward the $551 \alpha$-phosphate and stabilizes the planar conformation of the $\mathrm{P}_{\alpha} \mathrm{O}_{3}$ 552 group. In addition, the length of the hydrogen bond between $553 \mathrm{~K} 95$ and the $\mathrm{O}_{3 \beta}{ }^{\text {UTP }}$ oxygen is decreased in the transition state, 554 providing more stability to the position of the $\beta$-phosphate. 555 The tightening of the contacts among K380, K95, and the 556 phosphate moiety of UTP enhances the affinity of the enzyme 557 for the transition state and helps to compensate for the 558 increased atomic charges in the penta-coordinated intermedi559 ate. The positions of other residues, including H191 that 560 coordinates the Glc-1-P phosphate, remain unchanged between 561 the transition and prereactive states. Together, these results 562 show that strictly conserved positively charged residues in the 563 active site contribute to catalysis via electrostatic stabilization of 564 the transition state, consistent with the loss of activity in K95A 565 and K380A mutants. ${ }^{19}$

566 In agreement with an $\mathrm{S}_{\mathrm{N}} 2$ associative mechanism, the 567 formation of a penta-coordinated intermediate is followed by 568 the stereochemical inversion of the $\alpha$-phosphate, the breaking 569 of the $\mathrm{P}_{\alpha}^{\text {UTP }}-\mathrm{O}_{3 \alpha}$ UTP bond, and generation of a leaving 570 pyrophosphate group and UDP-Glc. The resulting theoretical 571 model of the postreactive UGP.UDP-Glc $\cdot \mathrm{Mg}^{2+} \cdot \mathrm{PP}_{\mathrm{i}}$ complex 572 and its crystal structure show close similarity (r.s.m.d. 1.2 ̊) 573 (Figure 1E). According to the model, the pyrophosphate binds 574 into a pocket created by the NB loop residues G84-T87. 575 There, it is coordinated by hydrogen bonds with the main-chain 576 atoms of L85 and G86, the side chain of K95, and a number of 577 van der Waals interactions. In the crystal structure, the 578 positions of the $\gamma$ - and $\beta$-phosphates are occupied by the 579 sulfate ion and a water molecule, respectively. In both 580 experimental and theoretical models, $\mathrm{Mg}^{2+}$ is coordinated 581 between the $\alpha, \beta$, and $\gamma$ phosphates or their analogs in a similar 582 way. Inversion of the $\alpha$-phosphate and separation of $\mathrm{PP}_{\mathrm{i}}$ induce 583 small shifts of the H191, K380, and K95 side chains and the 584 main chain of the NB loop. The frontier molecular orbitals in 585 this state are localized on $\mathrm{O}_{3 \alpha}{ }^{\mathrm{PPi}}$ (HOMO) and $\mathrm{P}_{\alpha}{ }^{\text {UDP-Glc }}$ 586 (LUMO), which also represents the prereactive state of the 587 backward reaction (Figure $1 \mathrm{E}$ ). The distance between $\mathrm{O}_{3 \alpha}{ }^{\text {PPi }}$ 588 and $\mathrm{P}_{\alpha}$ UDP-Glc is $0.5 \AA$ longer compared with the reacting atoms 589 of the UGP.UTP.Glc-1-P complex. The reaction pathway 590 calculations predict the free energy barriers for the forward and 591 backward reactions to be 20.8 and $22.2 \mathrm{kcal} / \mathrm{mol}$, respectively 592 (Supporting Information Figure S3). The corresponding values 593 derived from the experimental specific activities $V_{\max }$ at a 594 physiological temperature of $37{ }^{\circ} \mathrm{C}$ (Supporting Information 595 Figure S2A,B) are 20.4 and $20.6 \mathrm{kcal} / \mathrm{mol}$ (experimental details 596 are given in the Supporting Information). Thus, the reaction 597 energy parameters produced in the calculated model are in a 598 good agreement with the experimental kinetics data and 599 reproduce the observed trend for the lower activation barrier in 600 the forward reaction.
Our study demonstrates that the interaction of K95 with the 601 $\beta$-phosphate is important to direct the pyrophosphate to its exit 602 channel.

\section{DISCUSSION}

604

The data presented in this manuscript filled critical gaps in the 605 knowledge of the LmUGP mechanism and allowed a complete 606 reconstruction of the enzymatic cycle. In the forward reaction, 607 the working cycle of LmUGP consists of five stages: closing 608 (activated by UTP binding), locking (initiated by Glc-1-P 609 binding), the enzyme-catalyzed chemical reaction, unlocking 610 (activated by pyrophosphate dissociation), and opening 611 (coupled with UDP-Glc dissociation).

612

LmUGP closing is activated by UTP, whose binding site 613 already exists in the open state. The primary role of the 614 nucleoside in UTP binding is supported by our structure of 615 LmUGP H191L mutant, and the crucial role of the UTP $\gamma$ - 616 phosphate in the activation mechanism is elucidated by the 617 structure of the LmUGP.dUpCpp complex. The activation 618 effect of the $\gamma$-phosphate involves the large shift of the NB loop, 619 which in its turn is bound to both the C-terminal domain and 620 the seven-stranded $\beta$-sheet (Figure 2B). This shift causes 621 conformational changes in these areas, particularly a torsional 622 deformation of the $\beta$-sheet. The latter leads to a formation of 623 the allosteric binding site for the second substrate. With this 624 definition of the role of the $\gamma$-phosphate in the allosteric 625 mechanism of LmUGP activation, our structure explains the 626 previous findings ${ }^{2}$ that UDP and UMP, lacking the $\gamma-627$ phosphate, are not able to activate LmUGP for the binding 628 of the second substrate, Glc-1-P.

Binding of the second substrate initiates further changes in 630 the LmUGP structure. The structural and kinetic data obtained 631 with the mutants E284A and L281D strongly suggest that the 632 UTP-induced torsional deformation of the $\beta$-sheet allows the 633 glucose ring to be prepositioned in its binding site so that the 634 interaction between the $\mathrm{O}^{\prime}$ and $\mathrm{O}^{\prime}$ ' hydroxyls of glucose and 635 the carboxyl of E284 can occur. This interaction pulls the 636 adjacent area of the handle, the SB loop and helix $\alpha 11$ toward 637 the C-terminal domain, which stabilizes the Glc-1-P binding 638 site, brings strictly conserved $\mathrm{H} 191$ and K380 to favorable 639 positions for phosphate binding, and closes the LmUGP 640 structure. Thus, E284 is playing a primary role in the process of 641 glucose stabilization by LmUGP (as reflected by the $>6$-fold 642 decrease in Glc-1-P affinity in the E284A mutant, Supporting 643 Information Figure S2D) while the SB loop plays a secondary 644 role. We defined this mechanism of the second substrate 645 stabilization as "the lock mechanism" and the residues R249- 646 Y302 as "the lock region" (Figure 1). The locking is facilitated 647 by the base of the handle, which acts as a transducer of tensile 648 force from E284 to the SB loop. The detailed allosteric 649 mechanism of the lock was revealed by molecular dynamics 650 simulations and confirmed by our kinetic and structural studies 651 of LmUGP mutants.

Torsional deformation of the $\beta$-sheet and conformational 653 changes in the lock area provide new insights into the 654 mechanism of specific recognition of the second substrate by 655 LmUGP. ${ }^{2}$ Superposition of the LmUGP.UDP-Glc $\cdot \mathrm{Mg}^{2+} \cdot \mathrm{SO}_{4} 656$ complex (postreactive state) with the LmUSP.UDP-Gal 657 complex $^{47}$ (a promiscuous UDP-sugar pyrophosphorylase) 658 showed that the main steric hindrance preventing the binding 659 of galactose by LmUGP is caused by the main chain of N306 660 $(\beta 11)$. N306 belongs to the area where the torsional 661 deformation of the $\beta$-sheet reaches its maximum. In LmUSP, 662 
663 the torsional deformation of the $\beta$-sheet is of a lesser 664 magnitude, which leaves more space to accommodate 665 unspecific sugars. This observation indicates that the specificity 666 for the second substrate in nucleotidyltransferases can be 667 controlled via allosteric mechanisms.

668 Binding and stabilization of both substrates brings LmUGP 669 to the most compact state optimal for the catalytic reaction. 670 Our ab initio QM/MM calculations of the LmUGP reaction 671 pathway displayed the chemical conditions for the start of the 672 reaction elucidated the role of each residue in the catalytic 673 center during the reaction and explained the observed necessity $674 \mathrm{of}^{\mathrm{Mg}^{2+}}$ for catalysis ${ }^{9}$ but not for the nucleotide binding. ${ }^{46}$ The 675 LmUGP catalysis involves the proximity and orientation effect 676 provided by the active site protein matrix, bond strain and 677 electrostatic activation by the magnesium cofactor, and 678 electrostatic stabilization of the transition state by the positively 679 charged residues K380 and K95. The latter is also playing an 680 important role in directing the pyrophosphate to its exit 681 channel. With this, we provide a molecular explanation for the 682 previously hypothesized role of the positively charged active 683 site residues in guiding the $\mathrm{PP}_{\mathrm{i}}$ release. ${ }^{11}$

684 The structural models and reaction free energy barriers for 685 the forward and backward reactions obtained from the QC 686 calculations were in a good agreement with experimental data. 687 The position of $\mathrm{Mg}^{2+}$ in the experimental postreactive state 688 structure and in the QC models of LmUGP is similar to that in 689 the structure of bacterial guanosine-diphospho-D-mannose 690 pyrophosphorylase (GMP) ${ }^{48}$ in complex with GTP (pdb code: $6912 \mathrm{X} 60$ ) and to one of the two observed $\mathrm{Mg}^{2+}$ positions in the 692 complex of UGP from C. glutamicum with UDP-Glc ${ }^{49}$ (pdb 693 code: 2PA4). It is interesting that in the latter structure as well 694 as in the complex of GMP with GDP-Man ${ }^{48}$ (pdb code: 2X5Z), 695 the catalytically important $\mathrm{Mg}^{2+}$ occupies another position that 696 coincides with the position of the K380 amino group in 697 LmUGP (Supporting Information Figure S4). Despite the 698 different position, the $\mathrm{Mg}^{2+}$ in all structures includes the $\alpha$ 699 phosphate group in its coordination sphere and imposes similar 700 distortions on the $\alpha$-phosphate geometry as defined in 701 LmUGP. On the basis of this finding, it can be concluded 702 that, independent of the binding mode of $\mathrm{Mg}^{2+}$ in the different 703 nucleotidyltransferases, its mechanism of substrate activation 704 remains the same.

705 The high-resolution crystal structure of the postreactive state 706 showed for the first time the binding sites of all UGP products 707 and allowed revealing the mechanism of unlocking the enzyme 708 after the reaction. The unlocking is initiated by dissociation of 709 the pyrophosphate and $\mathrm{Mg}^{2+}$ ions. This causes the destabiliza710 tion of the NB loop and the lock region as well as a 711 conformational change of the UDP-Glc phosphate moiety, thus 712 activating the structure for closed-to-open transition. The full 713 opening of the enzyme should then be coupled with the 714 dissociation of UDP-Glc with the glucose part dissociating first. $715 \mathrm{It}$ is of note to mention that we could confirm this reaction 716 sequence in a later structural study of human UGP (manuscript 717 in preparation).

718 The mechanisms of catalysis and allosteric regulation of 719 LmUGP as described in this work should significantly facilitate 720 investigations of the reaction cycles and allosteric regulation 721 networks of other nucleotidyltransferases that also follow 722 sequentially ordered bibi kinetics. Moreover, our data help 723 one to understand the coupling between global conformational 724 changes and events at the active site during the reaction of 725 these enzymes. This information would be particularly useful for drug-design applications utilizing nucleotidyltransferases as 726 drug targets. Structural similarities of nucleotidyltransferases 727 make targeting their active sites likely to cause unwanted, 728 unspecific disruption of enzymatic pathways in the host. A 729 solution to this problem can be provided by employing 730 allosteric effects in the drug-design strategy, utilizing the 731 conformational control by nonconserved residues located far 732 from the catalytic center. A comprehensive description of the 733 enzymatic mechanism and allosteric effects would thus be an 734 important prerequisite for rational development of highly 735 specific allosteric regulators for these enzymes. 736

Finally, our structural and kinetic data on the L281D mutant 737 as well as computational analysis reveal the mechanism of 738 mechanical control of the chemical reaction in the active site of 739 LmUGP. This mechanism involves the tensile force trans- 740 duction between the strictly conserved residue E284 and the SB 741 loop via the $\beta$-structure of the handle. Decreasing the efficiency 742 of the force transduction by disturbing the $\beta$-structure leads to 743 the loss in specific activity of the enzyme, as demonstrated by 744 the L281D mutation. This illustrates how regulating the 745 efficiency of mechanical force transduction in allosteric chains 746 could be used to alter the catalytic activity in allosteric enzymes. 747 Small molecule compounds producing similar effects could be 748 used in drug design and biotechnological applications. The 749 concept of mechanical regulation of enzymatic and homoge- 750 neous chemical catalysts has attracted attention as a result of its 751 potential applications in biotechnology ${ }^{50-52}$ and new functional 752 materials. ${ }^{53}$ Studying natural evolutionarily optimized mecha- 753 nisms of such regulation, as in the case of LmUGP, can have a 754 strong impact on the development of artificial mechanochem- 755 ical catalysts.

\section{ASSOCIATED CONTENT}

\section{S Supporting Information}

758

Crystallographic data and refinement statistics, experimental 759 electron density omit maps, kinetic data, reaction energy 760 diagram, and superposition of the active sites of nucleotidyl- 761 transferases. This information is available free of charge via the 762 Internet at http://pubs.acs.org/

\section{Accession Codes}

763 Coordinates for the structures LmUGPwt.UDP-Glc. Mg.SO ${ }_{4}, 765$ LmUGP $_{\text {L281D }} \cdot$ UDP-Glc, LmUGP $_{\mathrm{H} 191 \mathrm{~L}}$, and LmUGPwt. 766 dUpCpp have been deposited in the Protein Data Bank with 767 the accession codes 4M2A, 4M2B, 4J18, and 4M28, 768 respectively.

\section{AUTHOR INFORMATION}

770

\section{Corresponding Author}

771

*Phone: +49-5115323705. Fax: +49-5115325966. E-mail: 772 Fedorov.Roman@mh-hannover.de.

Present Address

773

${ }^{\dagger}$ Roche Diagnostics GmbH, Sandhofer Strasse 116, 68305775 Mannheim, Germany.

Notes

The authors declare no competing financial interest.

\section{ACKNOWLEDGMENTS}

We thank D. J. Manstein for continuous support and providing 780 research infrastructure, A. A. Granovsky and J. Kress for the 781 QM/MM version of Firefly Quantum Chemistry package and 782 their help, I. Chizhov and D. S. Chernavsky for helpful 783 discussions, and staff scientists at the synchrotron beamlines 784 
$785 \mathrm{X} 11$ and X13 at DESY/EMBL-Hamburg and beamlines ID23 786 and ID29 at the ESRF/Grenoble for their support during 787 diffraction data collection. This study received financial support 788 from the European Community's Seventh Framework Pro789 gramme (FP7/2007-2013; grant agreement No. 226716), the 790 German Research Foundation in the frame of the Cluster of 791 Excellence REBIRTH, and an intramural grant ("HiLF") of 792 Hannover Medical School. The funders had no role in study 793 design, data collection and analysis, decision to publish, or 794 preparation of the manuscript.

\section{$795 \square$ REFERENCES}

796 (1) Kuchta, K.; Knizewski, L.; Wyrwicz, L. S.; Rychlewski, L.; 797 Ginalski, K. Nucleic Acids Res. 2009, 37, 7701-7714.

798 (2) Lamerz, A. C.; Haselhorst, T.; Bergfeld, A. K.; von Itzstein, M.; 799 Gerardy-Schahn, R. J. Biol. Chem. 2006, 281, 16314-16322.

800 (3) Kleczkowski, L. A.; Martz, F.; Wilczynska, M. Phytochemistry $8012005,66,2815-2821$.

802 (4) Blankenfeldt, W.; Asuncion, M.; Lam, J. S.; Naismith, J. H. EMBO 803 J. 2000, 19, 6652-6663.

804 (5) Koropatkin, N. M.; Cleland, W. W.; Holden, H. M. J. Biol. Chem. 805 2005, 280, 10774-10780.

806 (6) Roeben, A.; Plitzko, J. M.; Korner, R.; Bottcher, U. M.; Siegers, 807 K.; Hayer-Hartl, M.; Bracher, A. J. Mol. Biol. 2006, 364, 551-560.

808 (7) Sivaraman, J.; Sauve, V.; Matte, A.; Cygler, M. J. Biol. Chem. 2002, 809 277, 44214-44219.

810 (8) Jin, X.; Ballicora, M. A.; Preiss, J.; Geiger, J. H. EMBO J. 2005, 24, $811694-704$.

812 (9) Turnquist, R. L.; Gillett, T. A.; Hansen, R. G. J. Biol. Chem. 1974, 813 249, 7695-7700.

814 (10) Barton, W. A.; Lesniak, J.; Biggins, J. B.; Jeffrey, P. D.; Jiang, J.; 815 Rajashankar, K. R.; Thorson, J. S.; Nikolov, D. B. Nat. Struct. Biol. 816 2001, 8, 545-551.

817 (11) Zuccotti, S.; Zanardi, D.; Rosano, C.; Sturla, L.; Tonetti, M.; 818 Bolognesi, M. J. Mol. Biol. 2001, 313, 831-843.

819 (12) Ballicora, M. A.; Iglesias, A. A.; Preiss, J. Photosynth. Res. 2004, $82079,1-24$.

821 (13) Martinez, L. I.; Piattoni, C. V.; Garay, S. A.; Rodrigues, D. E.; 822 Guerrero, S. A.; Iglesias, A. A. Biochimie 2011, 93, 260-268.

823 (14) Smith, T. L.; Rutter, J. Mol. Cell 2007, 26, 491-499.

824 (15) Ballicora, M. A.; Iglesias, A. A.; Preiss, J. Microbiol. Mol. Biol. Rev. 825 2003, 67, 213-225.

826 (16) Martz, F.; Wilczynska, M.; Kleczkowski, L. A. Biochem. J. 2002, 827 367, 295-300.

828 (17) Macrae, J. I.; Obado, S. O.; Turnock, D. C.; Roper, J. R.; 829 Kierans, M.; Kelly, J. M.; Ferguson, M. A. Mol. Biochem. Parasitol. 830 2006, 147, 126-136.

831 (18) Urbaniak, M. D.; Tabudravu, J. N.; Msaki, A.; Matera, K. M.; 832 Brenk, R.; Jaspars, M.; Ferguson, M. A. J. Bioorg. Med. Chem. Lett. 833 2006, 16, 5744-5747.

834 (19) Steiner, T.; Lamerz, A. C.; Hess, P.; Breithaupt, C.; Krapp, S.; 835 Bourenkov, G.; Huber, R.; Gerardy-Schahn, R.; Jacob, U. J. Biol. Chem. 836 2007, 282, 13003-13010.

837 (20) Damerow, S.; Lamerz, A. C.; Haselhorst, T.; Fuhring, J.; 838 Zarnovican, P.; von Itzstein, M.; Routier, F. H. J. Biol. Chem. 2010, $839285,878-887$.

840 (21) Kabsch, W. Acta Crystallogr., Sect. D: Biol. Crystallogr. 2010, 66, 841 125-132.

842 (22) Winn, M. D.; Ballard, C. C.; Cowtan, K. D.; Dodson, E. J.; 843 Emsley, P.; Evans, P. R.; Keegan, R. M.; Krissinel, E. B.; Leslie, A. G.; 844 McCoy, A.; McNicholas, S. J.; Murshudov, G. N.; Pannu, N. S.; 845 Potterton, E. A.; Powell, H. R.; Read, R. J.; Vagin, A.; Wilson, K. S. 846 Acta Crystallogr., Sect. D: Biol. Crystallogr. 2011, 67, 235-242.

847 (23) Navaza, J. Acta Crystallogr., Sect. A: Found. Crystallogr. 1994, 848 A50, 157-163.

849 (24) Brunger, A. T. Nat. Protoc. 2007, 2, 2728-2733.

850 (25) Brunger, A. T.; Adams, P. D.; Clore, G. M.; DeLano, W. L.; 851 Gros, P.; Grosse-Kunstleve, R. W.; Jiang, J. S.; Kuszewski, J.; Nilges,
M.; Pannu, N. S.; Read, R. J.; Rice, L. M.; Simonson, T.; Warren, G. L. 852 Acta Crystallogr., Sect. D: Biol. Crystallogr. 1998, 54, 905-921. 853

(26) Sheldrick, G. M. Acta Crystallogr., Sect. A: Found. Crystallogr. 854 2008, 64, 112-122.

(27) Murshudov, G. N.; Vagin, A. A.; Dodson, E. J. Acta Crystallogr., 856 Sect. D: Biol. Crystallogr. 1997, 53, 240-255.

(28) Perrakis, A. Sixma, T. K; Wilson, K. S, Lamzin, V. S. A Crystallogr., Sect. D: Biol. Crystallogr. 1997, 53, 448-455. 859

(29) Emsley, P.; Lohkamp, B.; Scott, W. G.; Cowtan, K. Acta 860 Crystallogr., Sect. D: Biol. Crystallogr. 2010, 66, 486-501. 861

(30) Schroder, G. F.; Levitt, M.; Brunger, A. T. Nature 2010, 464, 862 $1218-1222$

(31) Granovsky, A. A. Firefly; 2009.

863

(32) Schmidt, M. W.; Baldridge, K. K.; Boatz, J. A.; Elbert, S. T.; 865 Gordon, M. S.; Jensen, J. H.; Koseki, S.; Matsunaga, N.; Nguyen, K. A.; 866 Su, S.; Windus, T. L.; Dupuis, M.; Montgomery, J. A. J. Comput. Chem. 867 1993, 14, 1347-1363.

(33) Becke, A. D. J. Chem. Phys. 1993, 98, 5648-5652.

(34) Lee, C.; Yang, W.; Parr, R. G. Phys. Rev. B: Condens. Matter 870 Mater. Phys. 1988, 37, 785-789.

(35) Vosko, S. H.; Wilk, L.; Nusair, M. Can. J. Phys. 1980, 58, 1200- 872 1211.

(36) Stephens, P. J.; Devlin, F. J.; Chabalowski, C. F.; Frisch, M. J. J. 874 Phys. Chem. 1994, 98, 11623-11627. 875

(37) Lii, J. H.; Allinger, N. L. J. Am. Chem. Soc. 1989, 111, 8566- 876 8575 .

(38) Allinger, N. L.; Yuh, Y. H.; Lii, J. H. J. Am. Chem. Soc. 1989, 111, 878 $8551-8566$.

(39) Lii, J. H.; Allinger, N. L. J. Am. Chem. Soc. 1989, 111, 8576- 880 8582.

(40) Baker, J. J. Comput. Chem. 1986, 7, 385-395.

(41) Helgaker, T. Chem. Phys. Lett. 1991, 182, 503-510.

(42) Culot, P.; Dive, G.; Nguyen, V. H.; Ghuysen, J. M. Theor. Chim. 884 Acta 1992, 82, 189-205.

(43) Gonzalez, C.; Schlegel, H. B. J. Chem. Phys. 1989, 90, 2154- 886 2161.

(44) Hine, J. Adv. Phys. Org. Chem. 1977, 15, 1-61.

(45) Mulliken, R. S. J. Chem. Phys. 1955, 23, 1833-1840.

(46) Stevens, R. A. J.; Phelps, C. F. Biochem. J. 1976, 159, 65-70. 890

(47) Dickmanns, A.; Damerow, S.; Neumann, P.; Schulz, E. C.; 891 Lamerz, A. C.; Routier, F. H.; Ficner, R. J. Mol. Biol. 2011, 405, 461- 892 478.

(48) Pelissier, M. C.; Lesley, S. A.; Kuhn, P.; Bourne, Y. J. Biol. Chem. 894 2010, 285, 27468-27476.

(49) Thoden, J. B.; Holden, H. M. Protein Sci. 2007, 16, 1379-1388 896

(50) Choi, B.; Zocchi, G.; Wu, Y.; Chan, S.; Jeanne, P. L. Phys. Rev. 897 Lett. 2005, 95, 078102.

(51) Saghatelian, A.; Guckian, K. M.; Thayer, D. A.; Ghadiri, M. R. J. 899 Am. Chem. Soc. 2003, 125, 344-345. 900

(52) Choi, B.; Zocchi, G.; Canale, S.; Wu, Y.; Chan, S.; Perry, L. J. 901 Phys. Rev. Lett. 2005, 94, 038103.

(53) Piermattei, A.; Karthikeyan, S.; Sijbesma, R. P. Nat. Chem. 2009, 903 $1,133-137$. 